\title{
Análisis del comportamiento estadístico y aproximación fractal en la recaudación del impuesto sobre nóminas y asimilados en el estado de Quintana Roo
}

Sergio Lagunas Puls Universidad del Caribe, México

slagunas@ucaribe.edu.mx

\section{Genaro Aguilar}

Gutiérrez.

Instituto Politécnico Nacional gaguilarg@ipn.mx

José Juan Rodríguez

Trejo

Universidad del Caribe, México 100300236@ucaribe.edu.mx

\section{Resumen}

Este trabajo analiza la recaudación del impuesto sobre nóminas y asimilados en el estado de Quintana Roo de 2000 a 2010. La aportación principal es identificar el comportamiento estadístico recaudatorio mediante la aproximación fractal, estableciendo patrones iterativos en el nivel estatal, municipal, por la totalidad de la serie analizada de 132 meses y en periodos menores, sirviendo como detonante para aplicarse en otras localidades y gravámenes con el fin de profundizar en algunas características de la fiscalidad. Dos criterios estadísticos fueron empleados para determinar la normalidad, o no, de las series de recaudación fiscal municipal en el periodo de estudio; dichos criterios son el de la regla empírica y el que utiliza parámetros del teorema de Chebyshev; como contraste, se aplicó la prueba Jarque-Bera a los residuos, concluyendo que la recaudación por ejercicio fiscal en Quintana Roo (con datos municipales) no sigue una distribución normal, razón por la cual se utilizó la aproximación fractal. Los resultados con datos de recaudación fiscal por municipios para ese estado muestran la presencia de un comportamiento fractal (repetitivo y cíclico) en la recaudación, lo que puede interpretarse como la presencia de una base fiscal consolidada, justificando así la conclusión de que hay fuerza (el nivel de recaudación observado) en las finanzas públicas locales. La misma conclusión se tiene, con diferentes cortes temporales, al analizar y detectar presencia de comportamiento fractal específica para la recaudación observada en los municipios de Benito Juárez (Cozumel) y Othón P. Blanco, de 2000 a 2010.

Palabras clave: recaudación fiscal, pruebas de normalidad, aproximación fractal. 


\title{
Analysis of the statistical behavior and fractal approach in the collection of payroll tax on staff and non-staff in Quintana Roo
}

\begin{abstract}
This paper analyzes the tax collection from payroll tax on staff and non-staff in the state of Quintana Roo from 2000 to 2010. Its main contribution is to identify the statistical revenue behavior using a fractal approach, establishing iterative patterns at both state and municipal level, for the entire analyzed series of 132 months as well as shorter time periods. It serves as a trigger for application in other locations and charges in order to explore some features of taxation. Two statistical criteria were used to determine normal or abnormal series of municipal tax collection during the study period, namely, the Empirical Rule criteria and parameters using Chebyshev's theorem. As a contrast, the Jarque-Bera test was applied on residuals, concluding that the fiscal revenue from the state of Quintana Roo (municipal data) does not follow a normal distribution, which is why the fractal approach has been used. The results with data for tax collection by municipalities throughout Quintana Roo show the presence of a fractal behavior (repetitive and cyclic) in revenue, which can be interpreted as the presence of a consolidated tax base, thus justifying the conclusion that there is strength (the observed level of collection) in local public finances. The same conclusion is obtained, with different temporal cuts, when analyzing and detecting the presence of specific fractal revenue behavior observed in municipalities of Benito Juarez (Cozumel) and Othon P. Blanco from 2000 to 2010.
\end{abstract}

Keywords: tax collection, normality test, fractal approach

\section{Introducción}

La recaudación fiscal ha significado a lo largo de la historia un tema de relevante interés debido a la materialización de recursos públicos necesarios para pagar bienes y financiar programas de redistribución de la renta, situación que se aplica en los niveles de gobierno federal, estatal y municipal (Samuelson y Nordhaus, 2005).

Han existido interesantes propuestas en materia fiscal de nivel macroeconómico, destacando la propuesta de Arthur Betz Laffer (2004), la cual plantea que el incremento en las tasas de impuestos aumenta la recaudación sólo hasta cierto punto, ya que a partir del máximo óptimo un mayor incremento provoca una menor recaudación fiscal. Resulta interesante el análisis en la pérdida en peso muerto que puede ser generada por un sistema tributario, argumentando que a una menor tasa 
de impuesto los ingresos se incrementan con una tasa de impuesto adecuada o media, mientras que los ingresos tributarios se incrementan en una mayor medida; sin embargo, con una tasa impositiva demasiado alta, los ingresos tributarios sólo se incrementan en una pequeña parte, del resto se dice que es un espacio o pérdida en peso muerto (Mankiw, 2009).

En un esfuerzo por comprender el comportamiento en la recaudación fiscal local existen diversos estudios contemporáneos, destacando un análisis realizado en diez países latinoamericanos en donde se reconoce la importancia de la fiscalidad y su incidencia en el desarrollo económico territorial; no obstante, el grado de análisis no ha ido más allá que el de establecer comparativas en la normatividad, cobertura y tasas impositivas (Cuervo y Williner, 2009); por lo anterior, se identifica la necesidad de realizar análisis más profundos en las recaudaciones locales que permitan conocer el comportamiento de la recaudación fiscal. Resultó interesante iniciar con una orientación a la propuesta de la geometría fractal impulsada por Benoit Mandelbrot (2003), referente a la presencia constante de objetos y circunstancias con patrón iterativo y análisis enfocados a la economía a través del comportamiento de los mercados de valores (Peters, 1994; Mandelbrot y Hudson, 2006; Braun, 1994); se incluye a detalle el comportamiento de la recaudación fiscal en el estado de Quintana Roo, específicamente en lo que se refiere al Impuesto sobre Nóminas y Asimilados, concepto que representa el $47.46 \%$ del total de impuestos que figuran en la Ley de Ingresos para el estado de Quintana Roo para el ejercicio fiscal 2012 (Congreso del Estado, 2011). Asimismo, se desarrolla un análisis gráfico, donde se hacen presentes periodos con picos altos, repetitivos, en escalas distintas en el nivel estatal, municipal, inclusive con diferentes rangos y periodos.

\section{Composición de la recaudación fiscal estatal}

Conforme a la Ley de Ingresos del estado de Quintana Roo para el Ejercicio Fiscal de 2012 por concepto de impuestos se contempló recaudar 1257886 393, de los cuales el 597041831 corresponden al impuesto sobre nóminas y asimilables, lo que significa el $47.46 \%$ del total de ingresos por conceptos de impuestos.

El elemento fundamental para la investigación lo constituye la recaudación informada oficialmente y de la cual se desprenden los análisis numéricos y gráficos. 


\section{Cuadro $1^{1}$ \\ Composición de la recaudación del Impuesto sobre nóminas y asimilados: 2000-2010 (miles de pesos)}

\begin{tabular}{|c|c|c|c|c|c|c|c|c|c|c|c|}
\hline Municipio & 2000 & 2001 & 2002 & 2003 & 2004 & 2005 & 2006 & 2007 & 2008 & 2009 & 2010 \\
\hline $\begin{array}{l}\text { Othón. P. } \\
\text { Blanco }\end{array}$ & 12843 & 14090 & 19116 & 24604 & 28319 & 38412 & 49611 & 60511 & 66810 & 74909 & 81436 \\
\hline $\begin{array}{l}\text { Benito } \\
\text { Juárez }\end{array}$ & 91290 & 107592 & 115152 & 144188 & 143871 & 157943 & 189850 & 221748 & 255862 & 239804 & 249825 \\
\hline Cozumel & 10213 & 12082 & 13172 & 14389 & 16120 & 15068 & 16031 & 15144 & 14321 & 12707 & 14351 \\
\hline $\begin{array}{l}\text { Isla } \\
\text { Mujeres }\end{array}$ & 797 & 798 & 850 & 1077 & 1135 & 1172 & 1481 & 2012 & 2500 & 3555 & 4009 \\
\hline $\begin{array}{l}\text { Felipe } \\
\text { Carrillo } \\
\text { Puerto }\end{array}$ & 193 & 225 & 244 & 267 & 318 & 410 & 391 & 752 & 3150 & 1379 & 1529 \\
\hline $\begin{array}{l}\text { José María } \\
\text { Morelos }\end{array}$ & 84 & 95 & 97 & 123 & 133 & 143 & 228 & 161 & 185 & 396 & 542 \\
\hline $\begin{array}{l}\text { Lázaro } \\
\text { Cárdenas }\end{array}$ & 60 & 73 & 85 & 180 & 233 & 237 & 291 & 331 & 410 & 367 & 389 \\
\hline Solidaridad & 14999 & 20633 & 21233 & 27453 & 39339 & 45881 & 62255 & 71656 & 83522 & 75967 & 83012 \\
\hline
\end{tabular}

Fuente: Elaboración propia con datos de la Secretaría de Hacienda de Quintana Roo

Es importante mencionar que la información corresponde a los municipios que existían en los años referidos, haciendo la aclaración de que al año 2012 se agregarán los municipios de Tulum² y Bacalar en los informes de recaudación.

\section{Comportamiento del impuesto sobre nómina y asimilados}

En distintos análisis latinoamericanos enfocados a variables económicas (Gálvez, 2005), inspirados en las propuestas relativamente recientes de la geometría fractal de Mandelbrot (2003), establecen como eje fundamental determinar los estadísticos descriptivos de las series de tiempo de interés para obtener factores iterativos como uno de los elementos de inferencia al comportamiento fractal.

\footnotetext{
${ }^{1}$ La información original no fue expresada en miles, el haberla incluido de esta forma corresponde a razones de espacio y formato.

${ }^{2}$ El municipio de Tulum inició recientemente en el año 2009, cuya administración estuvo a cargo de un consejo municipal hasta mediados del 2011, mismo año en que inicia el municipio de Bacalar.
} 
La metodología para obtener las estadísticas consistió en tabular los datos por ejercicio fiscal, totalizando por meses. Para determinar la normalidad, o no, en la distribución se emplearon los siguientes criterios: mediante localizadores ${ }^{z}$ para determinar si los datos cumplían con los criterios de la regla empírica y del teorema de Chebyshev (Anderson, Sweeny y Williams, 2008). También se determinaron los cuartiles $Q_{1}, Q_{3}$ para establecer el rango intercuartílico (Triola, 2011) y medir la normalidad mediante los límites superior e inferior; asimismo, se analizaron los residuos mediante la prueba Jarque-Bera (Ciuiu, 2005) que determinará finalmente la existencia de colas gruesas y el impacto asimétrico y de curtosis; de igual forma, se realizó un análisis gráfico por periodos de diciembre a febrero del siguiente ejercicio a efectos de identificar patrones que evidenciaran interactividad en un primer paso con la información de nueve años en el nivel estatal; posteriormente, con el comportamiento de un municipio y, finalmente, gráficas de periodos de 14 meses de distintos años y municipios.

A continuación, a manera de ejemplo, se presenta el análisis correspondiente al ejercicio 2000, haciendo la aclaración de que para todos los años se replicó la misma forma tabular, determinando los datos de interés. 


\section{Cuadro 2}

Análisis de la normalidad en la distribución de datos mediante localizadores z: Regla empírica y teorema de Chebyshev

\begin{tabular}{|c|c|c|c|c|c|}
\hline Meses & Recaudación año 2000 & $z=\left(\frac{x-\mu}{\sigma}\right)$ & $-1 \leq z \leq 1$ & $-2 \leq z \leq 2$ & $-3 \leq z \leq 3$ \\
\hline ene-00 & 13722972.87 & 2.34 & 0 & 0 & 1 \\
\hline feb-00 & 8661666.20 & -1.82 & 0 & 1 & 1 \\
\hline mar-00 & 10038668.33 & -0.69 & 1 & 1 & 1 \\
\hline abr-00 & 10004686.55 & -0.71 & 1 & 1 & 1 \\
\hline may-00 & 10839532.85 & -0.03 & 1 & 1 & 1 \\
\hline jun-00 & 10783860.07 & -0.07 & 1 & 1 & 1 \\
\hline jul-00 & 11528061.20 & 0.54 & 1 & 1 & 1 \\
\hline ago-00 & 10561200.91 & -0.26 & 1 & 1 & 1 \\
\hline sep-00 & 10991137.69 & 0.10 & 1 & 1 & 1 \\
\hline oct-00 & 10276770.97 & -0.49 & 1 & 1 & 1 \\
\hline nov-00 & 11437604.74 & 0.46 & 1 & 1 & 1 \\
\hline dic-00 & 11631319.19 & 0.62 & 1 & 1 & 1 \\
\hline & & \multirow[b]{3}{*}{$\begin{array}{l}\text { Por regla } \\
\text { empírica: }\end{array}$} & 10.00 & 11.00 & 12.00 \\
\hline & & & 0.83 & 0.92 & 1.00 \\
\hline & & & Normal & $\begin{array}{l}\text { Por teorema } \\
\text { Chebyshev }\end{array}$ & Normal \\
\hline
\end{tabular}

$$
\begin{array}{llll}
n=12 & & Q_{1}=\left(\frac{25}{100}\right) n= & 1^{\prime} 217245.31 \\
\mu=\frac{\sum x}{n}= & 10^{\prime} 873123.46 & Q_{3}=\left(\frac{75}{50}\right) n= & 1^{\prime} 460218.86 \\
\sigma=\sqrt{\frac{\sum(x-\mu)^{2}}{n-1}}= & 1^{\prime} 215845.45 & R I C=Q_{3}-Q_{1}= & 1^{\prime} 242973.55 \\
\sigma^{2}=\frac{\sum(x-\mu)^{2}}{n-1}= & 1.47828 \mathrm{E}+12 & L_{\text {inf }}=Q_{1}-1.5(R I C)=\mathbf{8}^{\prime} 352784.99 \\
& & L_{\text {sup }}=Q_{3}+1.5(R I C)=\mathbf{1 3}^{\prime} 324679.17
\end{array}
$$


Mediante los análisis por ejercicio se integra una síntesis de varianzas, desviaciones estándar, medias y puntos o localizadores ${ }^{z}$, también se indica el cumplimiento o no de la distribución normal y la presencia de datos atípicos.

\section{Cuadro 3}

\section{Síntesis de la normalidad y estadística descriptiva}

\begin{tabular}{|c|c|c|c|c|c|c|c|}
\hline \multirow[b]{2}{*}{ Año } & \multicolumn{3}{|c|}{ Prueba de normalidad } & \multirow[b]{2}{*}{$\mu=\frac{\sum x}{n}$} & \multirow[b]{2}{*}{$\sigma^{2}=\frac{\sum(x-\mu)^{2}}{n-1}$} & \multirow[b]{2}{*}{$\sigma=\sqrt{\frac{\Sigma(x-\mu)^{2}}{n-1}}$} & \multirow[b]{2}{*}{$R I C=Q_{x}-Q_{1}$} \\
\hline & $\begin{array}{l}\text { Regla } \\
\text { empírica }\end{array}$ & $\begin{array}{c}\text { Teorema } \\
\text { Chebyshev }\end{array}$ & $\begin{array}{c}\text { Datos } \\
\text { atípicos } \\
\text { por } \\
\text { límites }\end{array}$ & & & & \\
\hline 2000 & Sí & Sí & 1 & 10873123.46 & $1.47828 \mathrm{E}+12$ & 1215845.45 & 1242973.55 \\
\hline 2001 & No & Sí & 1 & 12965603.10 & $1.93512 \mathrm{E}+12$ & 1391084.57 & 1070472.88 \\
\hline 2002 & Sí & Sí & 1 & 14162504.78 & $2.65594 \mathrm{E}+12$ & 1629706.80 & 1693068.49 \\
\hline 2003 & Sí & Sí & 2 & 17690075.25 & $2.64938 \mathrm{E}+13$ & $2.64938 \mathrm{E}+13$ & 1399781.00 \\
\hline 2004 & Sí & Sí & 1 & 19122444.04 & $3.9038 \mathrm{E}+12$ & 1975803.93 & 1660254.50 \\
\hline 2005 & No & No & 0 & 21605459.83 & $1.27693 \mathrm{E}+13$ & 3573411.19 & 3884094.25 \\
\hline 2006 & Sí & Sí & 0 & 26678318.33 & $4.59337 \mathrm{E}+12$ & 2143214.33 & 3394843.25 \\
\hline 2007 & Sí & Sí & 2 & 31026237.92 & $1.19472 \mathrm{E}+13$ & 3456467.14 & 2175022.50 \\
\hline 2008 & Sí & Sí & 1 & 35563281.50 & $1.48106 \mathrm{E}+13$ & 3848452.37 & 4145127.00 \\
\hline 2009 & Sí & Sí & 2 & 34090447.92 & $3.94259 \mathrm{E}+13$ & 6279003.24 & 4017374.00 \\
\hline 2010 & Sí & Sí & 1 & 36257702.75 & $9.6151 \mathrm{E}+12$ & 3100822.91 & 3243211.25 \\
\hline
\end{tabular}

Como se observa, conforme a los criterios de la regla empírica, la cual implica que al menos el $68 \%$ de los datos estandarizados se encuentren a no más de una desviación estándar, $95 \%$ a no más de dos y el $99 \%$ dentro de tres desviaciones estándar, nueve de los once ejercicios analizados tendrían una distribución normal y dos no.

Con respecto a evaluar la normalidad mediante el criterio establecido en el teorema de Chebyshev, en donde el 75\% de los datos estandarizados deben estar dentro de dos desviaciones estándar y al menos el $89 \%$ se encuentren a no más de tres desviaciones estándar, diez de los once ejercicios tendrían una distribución normal.

Se decidió aplicar la prueba Jarque-Bera con el fin de analizar los residuos, teniendo como eje principal los valores para una distribución normal en donde la curtosis 
debe ser 3 y el sesgo adquirir un valor de 0 . En los casos en que la curtosis resultó mayor a 3 se establece la existencia de residuos con un comportamiento de cola pesada y en los resultados en donde se obtuvo un valor menor a 3 se determina un comportamiento liviano de los residuos.

El criterio utilizado en la prueba Jarque-Bera ${ }^{3}$ se estableció con base en lo que ha sido considerado en investigaciones precedentes (Ciuiu, 2005; Saporta, 1998; Gálvez, 2005) $)^{4}$, utilizando como elementos fundamentales los coeficientes de sesgo (s) y curtosis (c), por ejercicio individual y por el total de la series de tiempo. Se adoptan dos grados de libertad y con un nivel de confianza del 95\%, estableciendo como hipótesis nula el valor de 5.99 que correspondería a una curva normal. El cuadro 4 muestra la síntesis de los resultados por ejercicio.

\section{Cuadro 4}

\section{Evaluación de los residuos y aplicación de la prueba Jarque-Bera}

\begin{tabular}{c|c|c|c|c}
\hline Año & $\begin{array}{c}\text { Sesgo } \\
s=\frac{n}{(n-1)(n-2)} \sum\left(\frac{\boldsymbol{n}-\boldsymbol{\mu}}{\boldsymbol{\sigma}}\right)^{\mathbf{3}}\end{array}$ & $\begin{array}{c}\text { Curtosis } \\
c=\left[\frac{n(n+1)}{(n-1)(n-2)(n-3)} \sum\left(\frac{x-\mu}{s}\right)^{4}\right] \\
-\frac{\mathbf{3}(n-1)^{2}}{(n-2)(n-3)}\end{array}$ & $\begin{array}{c}\text { Jarque Bera } \\
j=\frac{n}{6}\left(s^{2}+\frac{1}{4}(c-3)^{2}\right)\end{array}$ & $\begin{array}{c}\text { Distribución } \\
\text { normal de } \\
\text { los residuos } \\
\text { (Sí / No) }\end{array}$ \\
\hline 2000 & 0.7131 & 2.5777 & 1.1063 & No \\
\hline 2001 & 1.5168 & 3.0119 & 4.6017 & No \\
\hline 2002 & 1.4893 & 3.6531 & 4.6494 & No \\
\hline 2003 & 2.7822 & 8.1243 & 28.6106 & No \\
\hline 2004 & 1.9420 & 5.3066 & 10.2027 & No \\
\hline 2005 & 0.3718 & 0.4504 & 3.5267 & No \\
\hline 2006 & -0.1876 & -0.9202 & 7.7544 & No \\
\hline 2007 & 1.6114 & 4.5881 & 6.4541 & No \\
\hline 2008 & 1.2368 & 1.1970 & 4.6846 & No \\
\hline 2009 & 1.1059 & 2.9364 & 2.4480 & No \\
\hline 2010 & 1.5164 & 2.8268 & 4.6137 & No \\
\hline
\end{tabular}

\footnotetext{
${ }^{3}$ En los estudios previos analizados fueron identificadas dos fórmulas que hacen referencia a la prueba Jarque Bera, comprobando la obtención de los mismos resultados en ambos casos. La otra opción distinta a la adoptada en el trabajo fue

$$
j b=n\left(\frac{s^{2}}{6}+\frac{(c-3)^{2}}{24}\right)
$$

${ }^{4}$ En el caso del trabajo de investigación de Gálvez (2005) se observó un planteamiento distinto.

$L M=\frac{N \cdot \text { sesgo }^{2}}{6}+\frac{N \cdot(\text { curtosis }-3)^{2}}{24}$
} 
Considerando los resultados en residuos, se rechaza la hipótesis nula estableciendo que en todos los ejercicios analizados, no se trataría de una distribución normal inclusive por los valores del sesgo y la curtosis, fortaleciendo la aproximación fractal debido a que la presencia de colas pesadas o irregularidad de su forma como la apreciación de Mandelbrot y otros autores (Domínguez Monterroza y Garzón, 2011), evidenciando la persistencia de residuos alejados de la centralidad y, además, contrastando con los estadísticos descriptivos y los criterios de regla empírica y teorema de Chebyshev.

El comportamiento estadístico evidencia también el mismo patrón obtenido en la mayoría de los ejercicios analizados individualmente, notando la presencia de un coeficiente Jarque-Bera de significancia para un comportamiento que no es normal.

$$
j b=\frac{n}{6}\left(s^{2}+\frac{1}{4}(c-3)^{2}\right)=7.827571071
$$

\section{Análisis gráfico e inferencia fractal}

La directriz principal fue localizar la presencia de evidencia repetitiva en la recaudación, en principio aceptando réplicas constantes aunque no correspondieran exactamente a una misma escala, tal como lo han argumentado distinguidos investigadores como Mandelbrot (2009) y Bouchaud y Potters (2000).

Se consideraron estudios previos aplicados a la economía, específicamente los enfocados al mercado de valores e índice de precios y cotizaciones como los realizados por Peters (1994) y Mandelbrot y Houdson (2006); en éstos la iteración e identificación de patrones definidos fueron básicos; además de que en general para el análisis gráfico fue directriz encontrar, en sentido intuitivo, una forma y la continuación de la misma en cualquier escala que arrojase el análisis.

En un primer momento se tuvo interés en visualizar el comportamiento del total de la serie de datos correspondiente a nueve años, de 2000 a 2010. El resultado fue un comportamiento repetitivo, cíclico, a manera de picos altos durante todos los periodos con la excepción del ejercicio 2005 en que sucedió el impacto del Huracán Wilma y del ejercicio 2009 por causas de la contingencia sanitaria por influenza H1N1. En la gráfica 1 se aprecia las réplicas encontradas. 


\section{Gráfica 1 \\ Representación de los picos altos como incidentes repetitivos en la recaudación nivel estado}

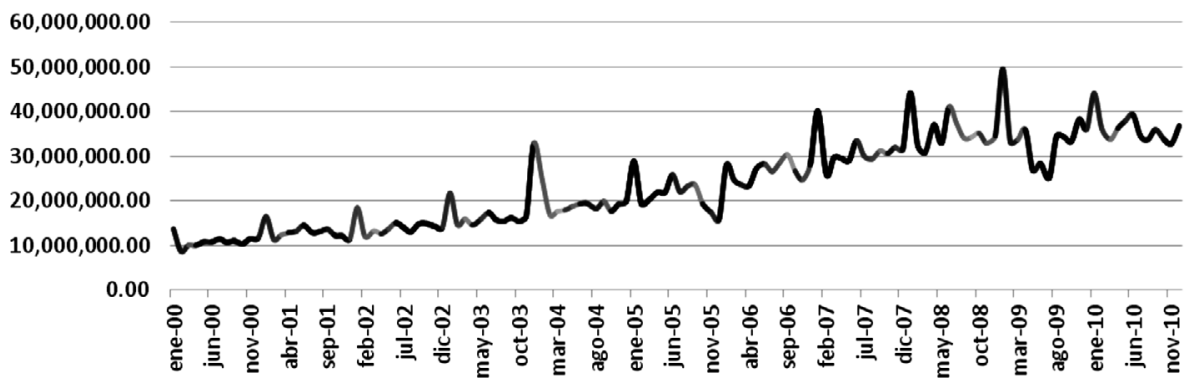

Al analizar visualmente la gráfica 1 es clara la presencia de réplicas en ciertos periodos, por lo que considerando la amplitud de la serie que incluye 132 meses con los datos oficiales de la recaudación existe sin lugar a dudas el criterio considerado por Mandelbrot como esencial de una situación fractal y los incrementos estacionarios que se presentan han sido considerados anteriormente (Embrechts y Maejima, 2002; Ramírez, Torres y Romero, 2012; Beran, 1994).

El siguiente punto de interés fue establecer si el comportamiento fractal además de estar presente en el nivel de la recaudación estatal también se encontraba en niveles inferiores, concretamente municipales, fueron tabulados los datos en los municipios de Benito Juárez, Cozumel y Othón P. Blanco, encontrando nuevamente la presencia fractal. 


\section{Gráfica 2}

Representación de los picos altos como incidentes repetitivos en cinco años, municipio de Benito Juárez

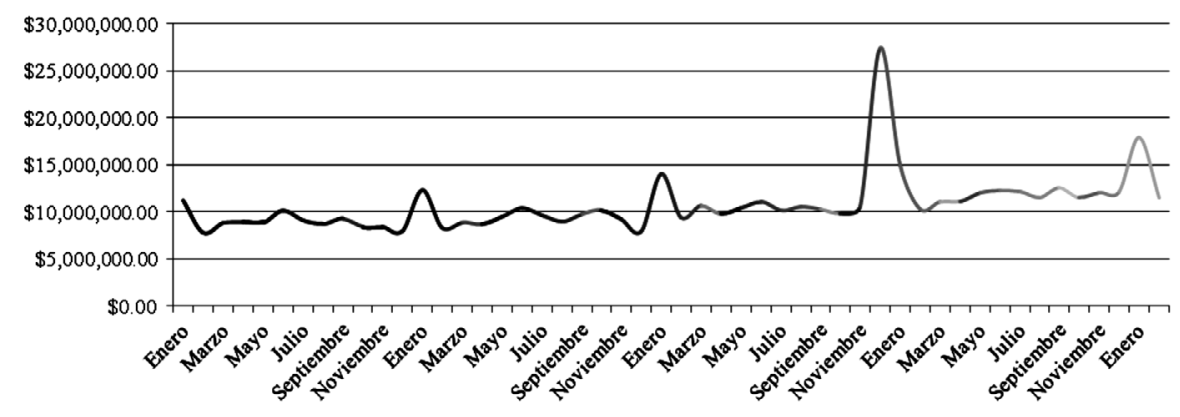

Para verificar lo anterior, se desarrollaron gráficas en periodos más cortos, de enero a febrero del siguiente año, en meses de años distintos y también diferentes periodos y municipios: Cozumel y Othón P. Blanco. En esta ocasión los datos fueron analizados mediante el programa Scientific Notebook que brinda una mejor reproducción en las líneas, los resultados nuevamente fueron imágenes replicadas en picos altos como se aprecia en las gráficas 3 y $4^{5}$.

\section{Gráfica 3}

\section{Representación de picos altos, municipio de Cozumel, enero 2002-febrero 2003}

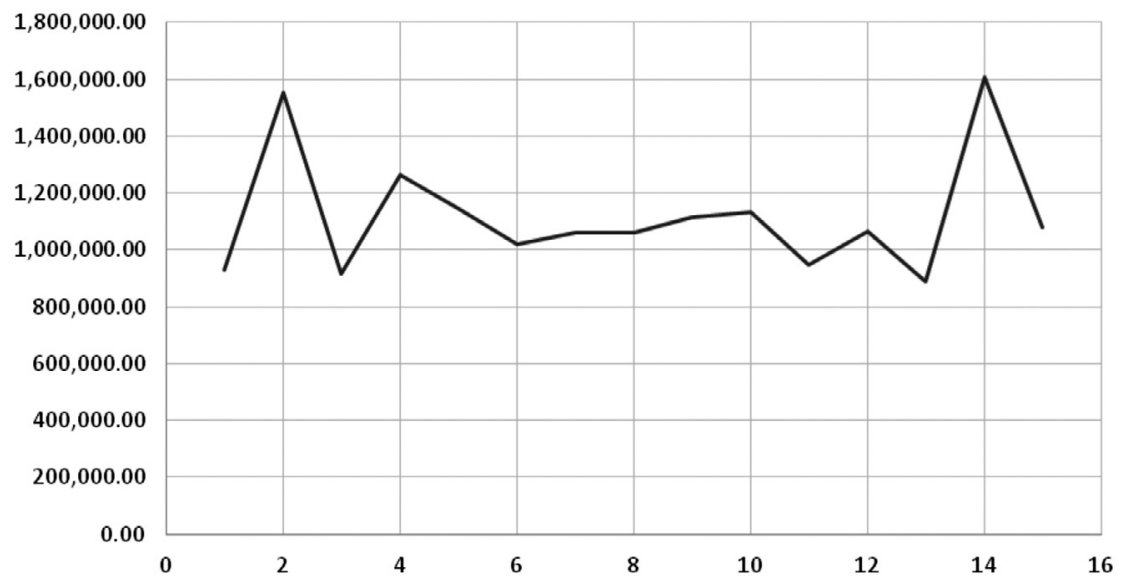

${ }^{5}$ Debido a la extensión del documento, únicamente se incluyen las gráficas de dos municipios: Benito Juárez y Othón P. Blanco 


\section{Gráfica 4}

\section{Representación de picos altos, municipio de Othón P. Blanco, enero 2004-febrero 2005}

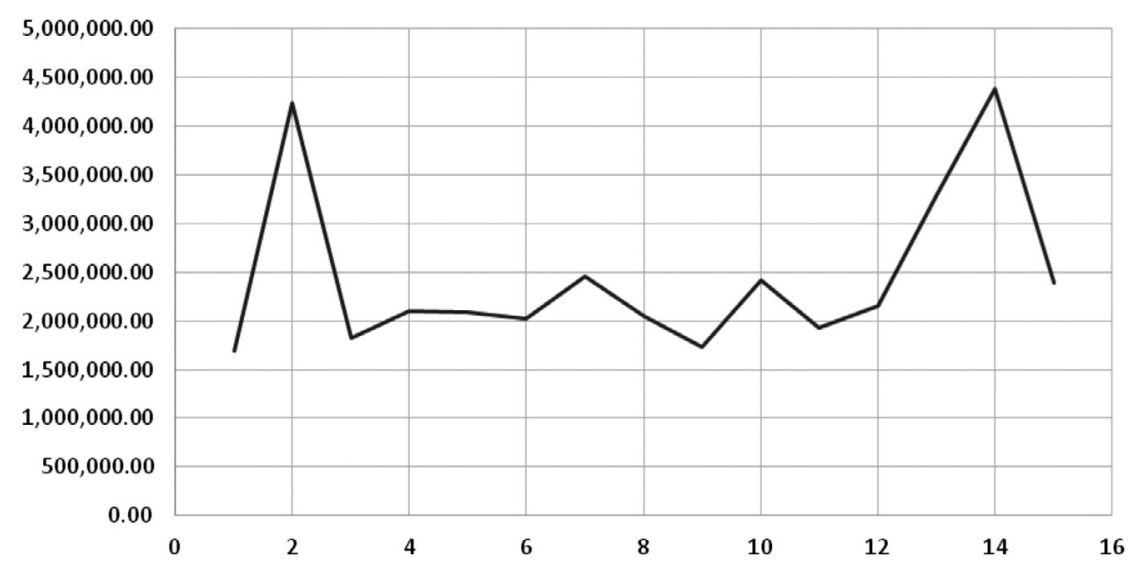

Finalmente, pretendiendo cumplir con la característica del grado de irregularidad e interrupción de la fractalidad y su reconocimiento a modelos cada vez más realistas (Mandelbrot, 2009) se determina la dimensión fractal mediante el software Benoit, versión 1.31, realizando cálculos correspondientes a los parámetros de autosimilitud con cuatro diferentes métodos ${ }^{6}:$ box dimension, perimeter-area dimension, information dimension y mass dimension. Para llevar a cabo lo anterior fue necesario realizar representaciones gráficas que incluyeron 132 observaciones correspondientes a la recaudación por cada mes; posteriormente, las gráficas se transformaron al formato de imagen con la extensión $b m p$, lo que hizo posible aplicar los distintos métodos para dimensión fractal y obtener las ecuaciones correspondientes. Los resultados se presentan en el siguiente cuadro:

${ }^{6}$ Se consideró pertinente la denominación común de los métodos en idioma inglés. 


\section{Cuadro 5}

\section{Parámetros de auto similitud en la recaudación del impuesto sobre nóminas 2000-2010}

\begin{tabular}{l|c|l}
\hline \multicolumn{1}{c|}{ Método } & Dimensión fractal & Ecuación obtenida \\
\hline Box dimension & 1.94388 & $y=3.52 e+05 x^{-1.95}$ \\
\hline Perimeter-area dimension & 1.01192 & $y=0.0815 x^{1.95}$ \\
\hline Information dimension & 1.97040 & $y=3.53 e+05 x^{-1.97}$ \\
\hline Mass dimension & 1.95845 & $y=3.7 x^{1.958}$ \\
\hline
\end{tabular}

Fuente: Elaboración propia con apoyo del programa Benoit versión 1.31

\section{Conclusiones}

Las pruebas de normalidad tradicionales con enfoque al teorema del límite central, como el criterio de regla empírica y los parámetros del teorema de Chebyshev, aplicadas a la recaudación fiscal analizada, son insuficientes para establecer enfáticamente la normalidad, ya que mediante los análisis a los residuos por coeficiente de asimetría y curtosis, calculados en lo individual y como insumos en la prueba de Jarque-Bera, sugieren que no necesariamente existe un comportamiento normal en la distribución del impuesto sobre nóminas y asimilado a salarios. La anterior situación evidenciaría la necesidad de incluir preguntas de investigación en los estudios fiscales similares realizados en otras entidades federativas; es decir, evitando asumir un comportamiento normal para el impuesto sobre nóminas.

Considerando los resultados en las estadísticas para los residuos, se estaría frente a la característica planteada por Mandelbrot con respecto a la irregularidad, cumpliendo también con la otra característica de autosimilitud obtenida en la gráfica, con que se concluye que el comportamiento fractal se encuentra presente.

El análisis también gráfico, aplicado a la totalidad de la serie de tiempo, que incluyó 132 observaciones mensuales, arrojó un patrón de evidente iteración, a excepción del año 2005 en que aconteció el embate del huracán Wilma y del año 2009 en que tuvo lugar la contingencia sanitaria para el virus H1N1; esta situación reforzaría algunos planteamientos de la teoría del caos (Prigogine, 2009) en donde un elemento de entrada puede causar múltiples situaciones distintas al patrón del orden común. 
Posteriormente a la distorsión gráfica que se observa en los ejercicios 2005 y 2009, la gráfica de forma paulatina, pero también determinante, retoma el comportamiento observado de los denominados picos altos en periodos muy específicos en cada año, normalmente en los meses de enero.

Al tratar de localizar evidencia repetitiva en la recaudación fiscal, además del nivel estatal constatado, se indagó en el comportamiento gráfico para los cuatro municipios más importantes del estado de Quintana Roo, encontrando iteración constante pese a la distorsión de incidencias negativas en 2005 y 2009.

Los incrementos en la recaudación observados en la totalidad de la serie, que correspondió a los once ejercicios fiscales, no son motivo para descartar la iteración y el proceso de autosimilitud, conclusión apoyada por lo dicho en otros planteamientos que reconocen la existencia de incrementos estacionales en un proceso de autosimilitud (Embrechts y Maejima, 2002; Ramírez, Torres y Toral, 2012).

Si bien se han realizado diversos estudios de tipo fractal con aplicación a las finanzas, la mayoría se ha orientado al índice de precios y cotizaciones (Chorofas, 1999; Ludlow, 1997; Peters, 1994); sin embargo, es importante hacer extensivas las aplicaciones al campo de la contaduría y en especial a la recaudación fiscal ya que - debido al comportamiento mostrado en el presente artículo, con énfasis en la iteración y los periodos que en gráficas representaron picos altos- debería ser parte de los criterios para formular la política fiscal en cada entidad, previendo excesos de asignación de recursos y posteriores limitantes presupuestales a las dependencias y entidades, procurando la estabilidad en el logro de programas específicos.

\section{Referencias}

Anderson, D., D. Sweeney y T. Williams (2008). Estadística para administración y economía. México: Cengage Learning.

Beran, J. (1994). Statics for Long-Memory Processes. EE. UU.: Chapman \& Hall/CRC.

Bouchaud, J. y M. Potters (2000). Theory of financial risks: from statistical physics to risk management. Reino Unido: Cambridge University Press. 
Braun, E. (1994). Caos, fractales y cosas raras. México: Fondo de Cultura Económica. Colección: la Ciencia en México 150.

Chorafas, D. (1999). Chaos theory in the financial markets. Illinois: Irwin Professional Publishing.

Ciuiu, D. (2005). Une modalit'e d'eviter les centiles aux regions de confiance et aux tests statistiques. Analele Universit ${ }^{\circ} t_{s}$ ii din Craiova 32 (1): 98-105.

(2008). On the Jarque-Bera normality test. In Working Papers of Macroeconomic Modelling Seminar 081802. Institute for Economic Forecasting.

Cuervo, L. y A. Williner (2009). Políticas e instituciones para el desarrollo económico territorial. Revista Líder 15 (11): 57-83.

Domínguez Monterroza, A. y D. Garzón-Alvarado (2011). Comportamiento fractal espacial en la expansión de la distribución del flujo sanguíneo cerebral en Azheimer. Revista Cubana de Investigaciones Biomédicas 30 (3): 424-438.

Embrechts, P. y M. Maejima (2002). Selfsimilar processes. Princeton series in applied mathematics. New Jersey: Princeton University Press.

Gálvez, E. (2005). Análisis fractal del mercado de valores de México 1978-2004. Instituto Politécnico Nacional. Tesis doctoral del Programa Doctorado en Ciencias, Escuela Superior de Comercio y Administración, Unidad Santo Tomás.

Laffer, A. (2004). The laffer curve: past, present and future. Heritage Foundation Backgrounder 1765. Disponible en: http://www.heritage.org/research/ reports/2004/06/the-laffer-curve-past-present-and-future

Lagunas, S. (2011). Modelo fiscal para incentivar el desarrollo económico en Quintana Roo. Universidad Popular Autónoma del Estado de Puebla, Tesis doctoral del Programa Doctorado en Desarrollo Económico y Sectorial Estratégico. 
Ludlow, J. (1997). Modelos, pronósticos y volatilidad de las series de tiempo generadas en la bolsa mexicana de valores. México: Universidad Autónoma Metropolitana, Azcapotzalco.

Mandelbrot, B. (2003). La geometría fractal de la naturaleza. Barcelona: Tusquets Editores.

(2009). Los objetos fractales. Barcelona: Tusquets Editores.

$\mathrm{y}$ J. Hudson (2006). The misbehavior of markets: a fractal view of financial turbulence, Nueva York: Basic Books.

Mankiw, G. (2009). Principios de economía. 5a. ed. México: Cengage Learning Editores.

Peters, E. (1994). Fractal market analysis: applying chaos theory to investment and economics. Nueva York: J. Wiley

Prigoni, I. (2009). Las leyes del caos. España: Drakontos

Ramírez, J., D. Torres y H. Toral (2012). Distinguishing stationary/nonstationary scaling processes using wavelet tsallis q-entropies. Mathematical Problems in Engineering. Artículo ID 867042.

Samuelson, P. y W. Nordhaus (2006). Macroeconomía con aplicaciones a Latinoamérica. México: McGraw Hill.

Saporta, G. y S. Ben Ammou (1998). Sur la normalit’e asymptotique des valeurs propres en ACM sous l'hypoth 'ese d'ind'ependence des variables. Revue de Statistique Appliqu'ee 46 (3): 21-35.

Triola, M. (2009). Estadística, México: Pearson. 